\title{
Circular Formation Control of Multiagent Systems with Any Preset Phase Arrangement
}

\author{
Lina Jin $\mathbb{D}^{1},{ }^{1}$ Shuanghe Yu $\left(\mathbb{D},{ }^{2}\right.$ and Dongxu Ren ${ }^{2}$ \\ ${ }^{1}$ School of Computer and Communication Engineering, Liaoning Shihua University, Fushun, China \\ ${ }^{2}$ College of Marine Electrical Engineering, Dalian Maritime University, Dalian, China \\ Correspondence should be addressed to Shuanghe Yu; shuanghe@dlmu.edu.cn
}

Received 17 September 2017; Revised 4 December 2017; Accepted 6 December 2017; Published 1 February 2018

Academic Editor: Yongji Wang

Copyright (c) 2018 Lina Jin et al. This is an open access article distributed under the Creative Commons Attribution License, which permits unrestricted use, distribution, and reproduction in any medium, provided the original work is properly cited.

\begin{abstract}
This paper deals with the circular formation control problem of multiagent systems for achieving any preset phase distribution. The control problem is decomposed into two parts: the first is to drive all the agents to a circle which either needs a target or not and the other is to arrange them in positions distributed on the circle according to the preset relative phases. The first part is solved by designing a circular motion control law to push the agents to approach a rotating transformed trajectory, and the other is settled using a phase-distributed protocol to decide the agents' positioning on the circle, where the ring topology is adopted such that each agent can only sense the relative positions of its neighboring two agents that are immediately in front of or behind it. The stability of the closed-loop system is analyzed, and the performance of the proposed controller is verified through simulations.
\end{abstract}

\section{Introduction}

Imitating the collective behaviors that occur in nature, the distributed control of multiagent systems (MAS), such as multiple autonomous underwater vehicles (AUVs) and unmanned aerial vehicles (UAVs) [1-3], has attracted a great deal of attention in control and robotic communities $[4,5]$ and has been extensively explored with different settings, including consensus [6], formation control [7, 8], flocking [9], distributed sensor networks [10], rendezvous [11], and source seeking $[12,13]$, through coordinating multiple autonomous mobile agents.

As one of these fundamental problems, the patternforming problem has attracted a considerable amount of research interest, where the agents are required to cooperatively generate and maintain the desired geometric patterns to perform various teamwork tasks. Formation patterns are typically limited to a point (rendezvous), line (flocking), or circle. The circular formation is a design method for steering the agents to orbit around a target along a common circle, which provides a simple geometric shape to collect data with a desired spatial and temporal distribution. In the community of systems and control, research efforts have been devoted to the circle formation problem for multiagent systems modeled as single or double integrators [14-18] and unicycles [19-26] under different communication topologies. Circular motion has been studied in the scenario of cyclic pursuit with ring topology [14-23]. A collective circular motion is addressed with a jointly connected communication condition [24, 25]. Under all-to-all communication condition, the phase potentials are used for uniform phase arrangement of particles along a circle [26]. In the aforementioned works, all the agents can enclose a fixed or moving target with position, distance, or bearing measurements in an equally circular distribution manner. However, for some special robotic application occasions, uniform distribution is unable to meet the practical demands; for example, AUV formation detects the concentration of oil pollution and UAV formation performs special escort missions in a nonuniform distribution [16]. Only a few works have presented the distributed control laws for a group of agents to formulate any given phase arrangement on a circle, and it should be noted that the agents are restricted to move in the one-dimensional space of a circle $[27,28]$.

The problem of circular formation of multiple agents with any preset phase arrangement in the two-dimensional space is addressed in this paper. The contributions can be 


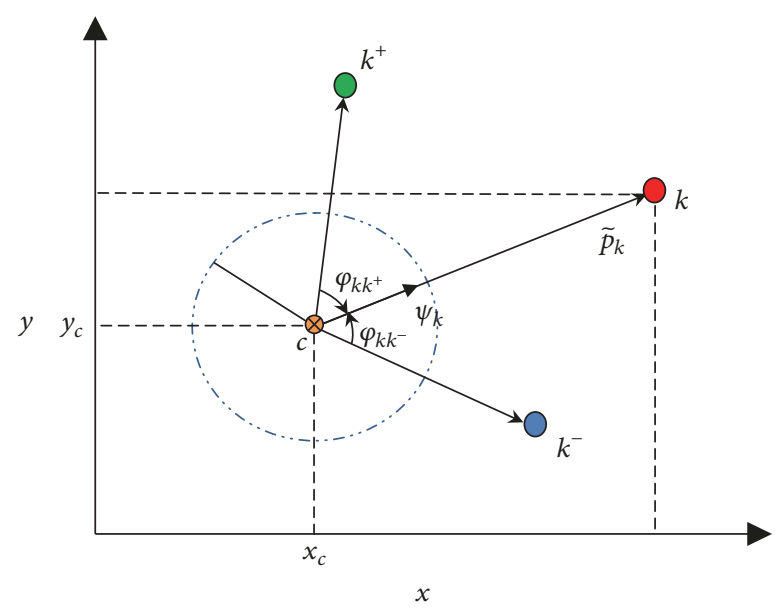

FIGURE 1: Agent, target, and the graphical view of notations.

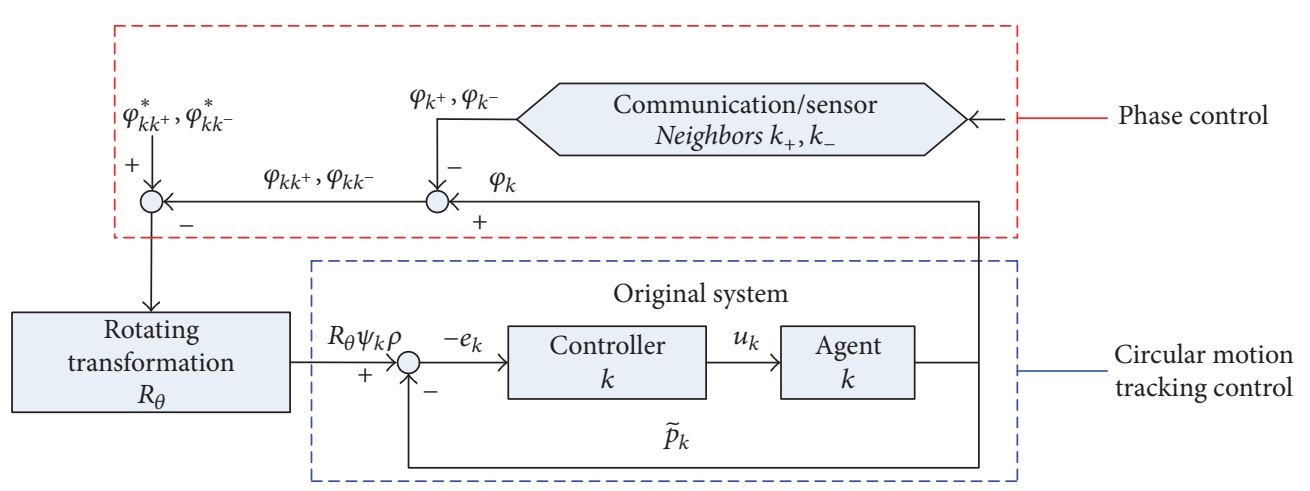

FIGURE 2: Feedback control design scheme of circular formation.

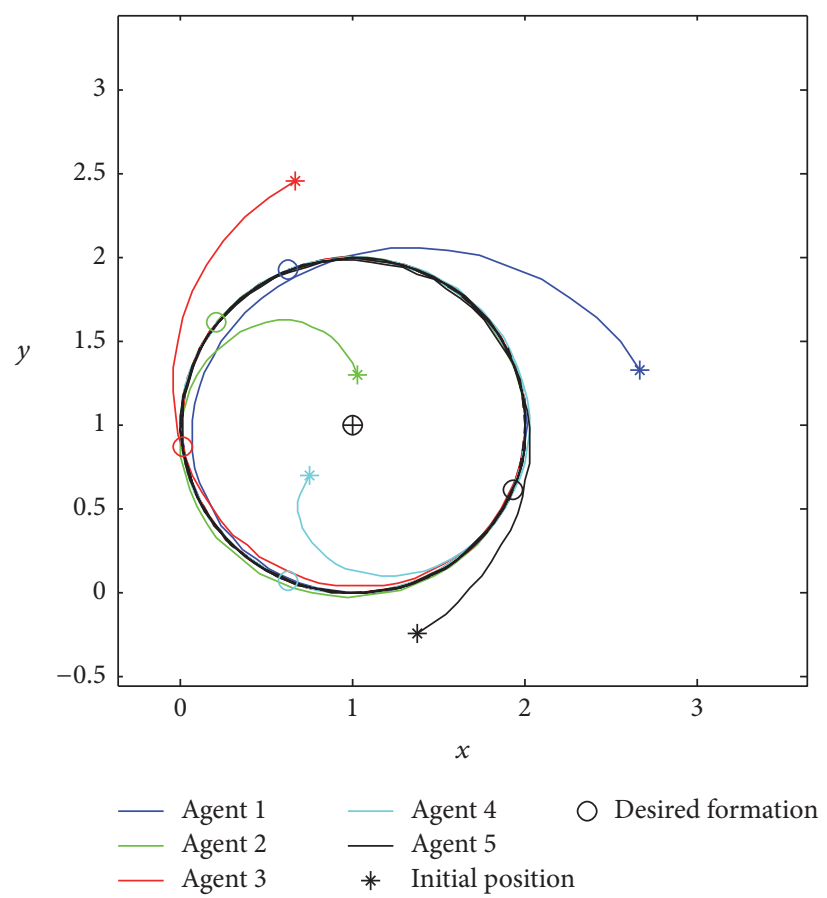

FIgURE 3: Circular formation trajectories $p_{k}(t)$ with a fixed target. 


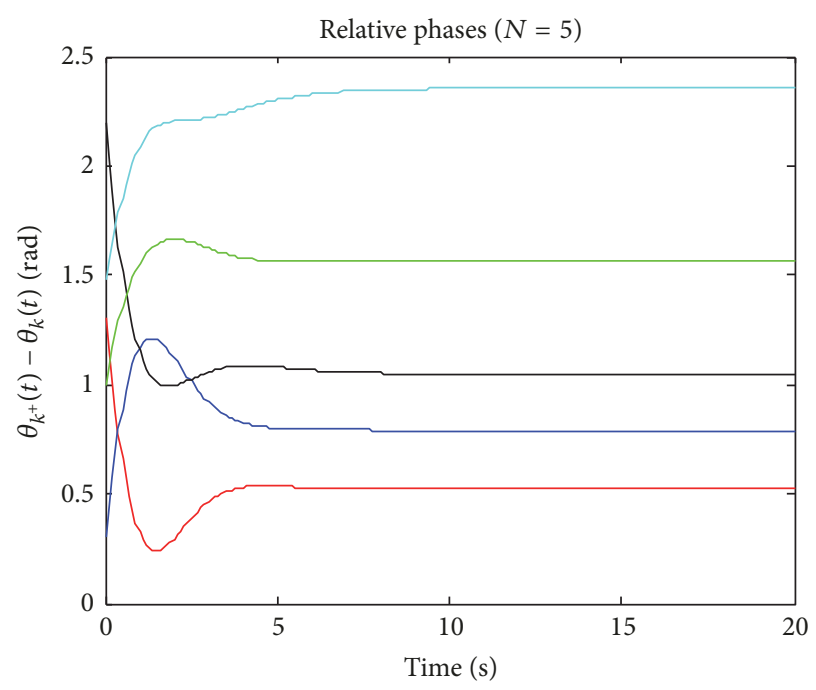

(a)

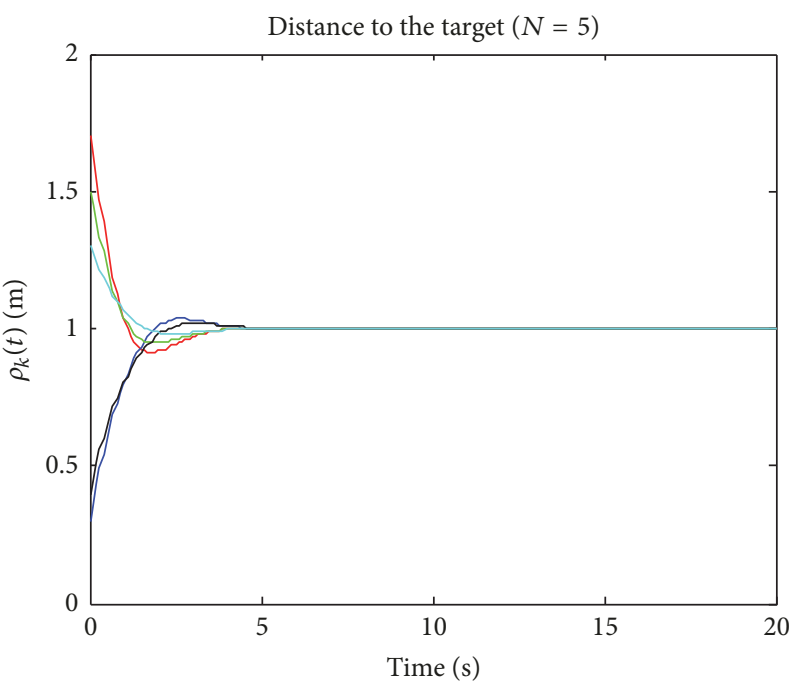

(b)

Figure 4: Trajectories with time of Figure 3. (a) Relative phases $\varphi_{k^{+} k}(t)$. (b) Distances to the target.

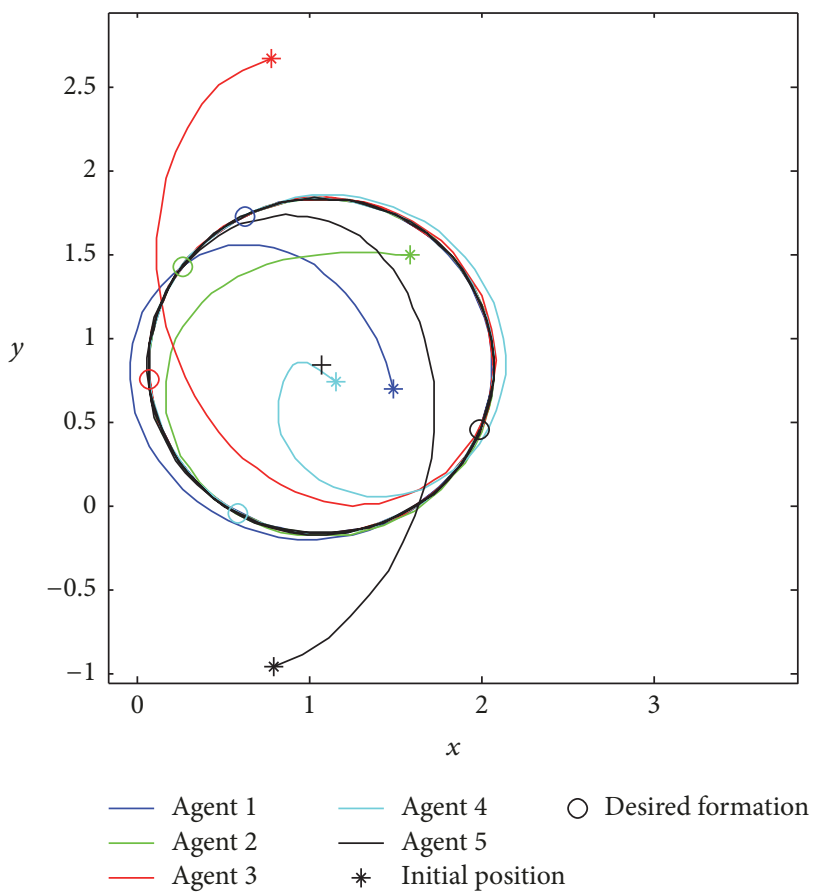

FIGURE 5: Circular formation trajectories $p_{k}(t)$ with center consensus.

summarized as follows. (1) Through introducing a rotated affine transformation, a tracking control strategy is proposed to achieve circular motion of agents by tracking a rotating matrix, where two cases of circling a target or not [29] are, respectively, considered. (2) Through combining the above control strategy with a multiagent phase cooperation mechanism, the circular formation task with any preset phase arrangement is implemented. Then, the phase arrangement algorithm without circle forming part in [28] is expanded to a two-dimensional model; that is, the positions of all the agents can be initialized out of the circle instead of being initialized on the assumed given circle. Furthermore, the advantage of order preservation is inherited because the circular motion control does not change the phase distribution during the entire motion. (3) An extension of the phase control law in [28] is presented to solve the positioning problem of the agents on the circle, such that some agents are located in the particular directions of the surrounded target according to the practical situation.

The paper is organized as follows. Some necessary preliminaries are presented and the control problem is formulated in Section 2. The tracking control law of circular motion is designed and analyzed in Section 3. Section 4 combines the above circular tracking control algorithm with a phase 


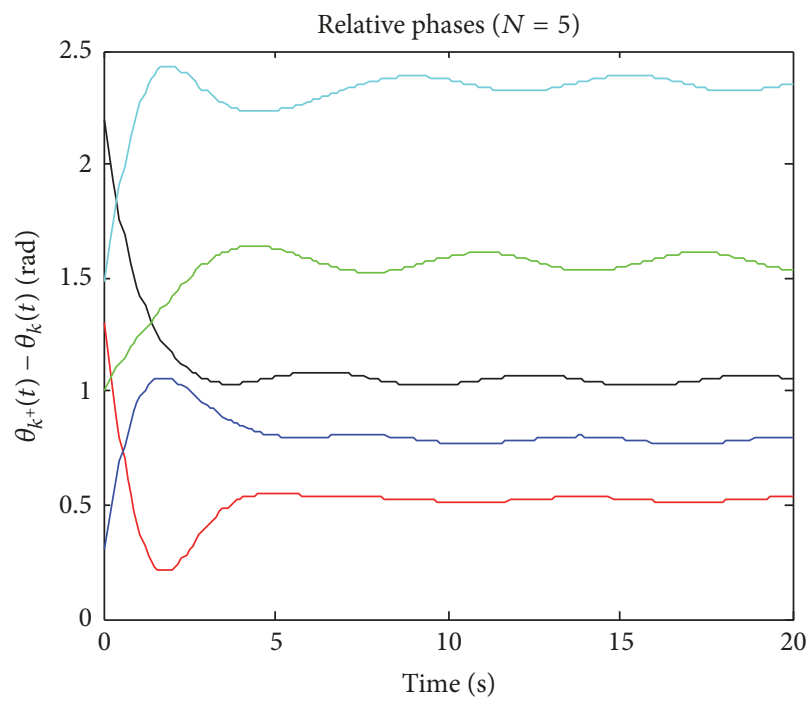

(a)
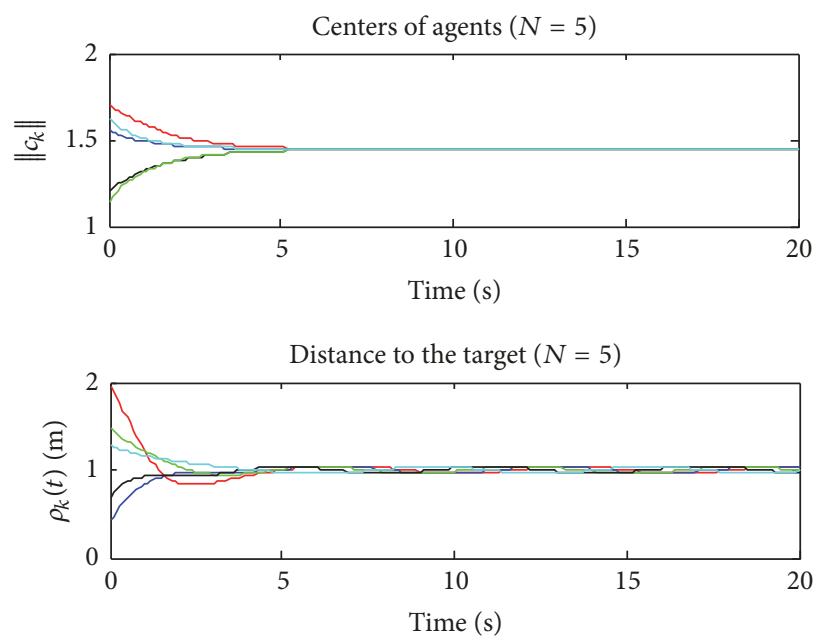

(b)

FIgURE 6: Trajectories with time of Figure 5. (a) Relative phases $\varphi_{k^{+}}(t)$. (b) Consensus center and distances to it.

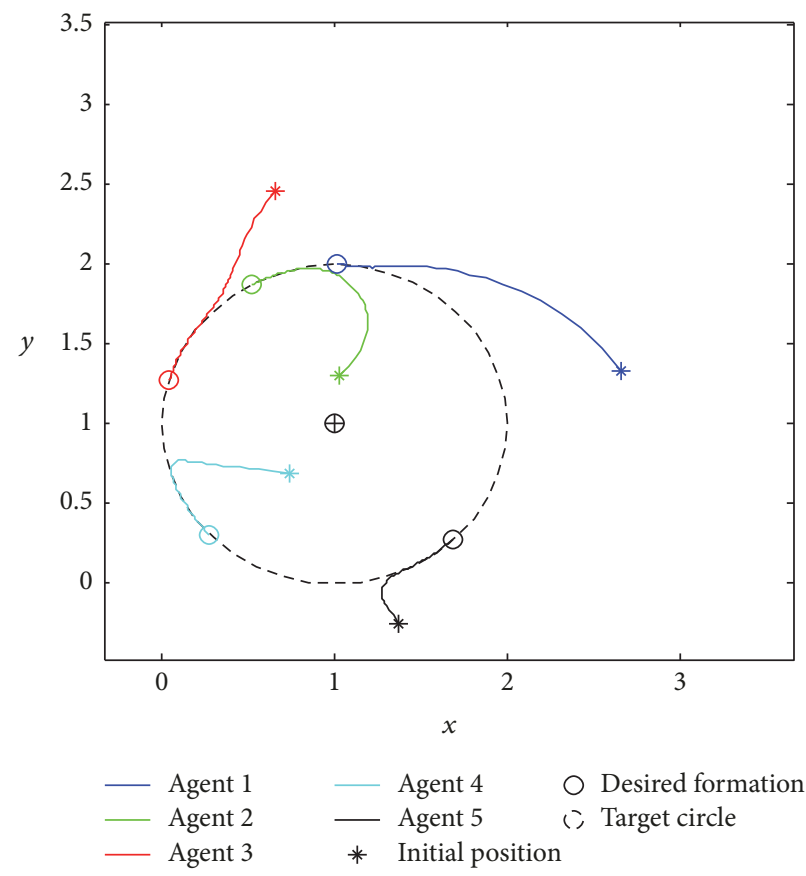

FIGURE 7: Circular formation trajectories $p_{k}(t)$ with $\varphi_{d}=\pi / 2$.

arrangement control law to achieve any phase arrangement along the circle. Simulation results given in Section 5 validate the strategy.

\section{Preliminaries and Problem Formulation}

2.1. Model of the Agents. Consider $N(N>$ integrator-modeled agents moving in the plane

2) single-

$$
\dot{p}_{k}(t)=u_{k}(t), \quad k=1, \ldots, N,
$$

where $p_{k}(t) \in \mathbb{R}^{2}$ and $u_{k}(t) \in \mathbb{R}^{2}$ denote the position and the control input of agent $k$, respectively. $P(t)=\left[p_{1}(t), \ldots, p_{N}(t)\right]^{T}$ denotes the stacked column vector. As shown in Figure 1, for a target with position $c \in \mathbb{R}^{2}$, $\tilde{p}_{k}(t)=p_{k}(t)-c$ denotes the relative displacement between agent $k$ in (1) and the target, $\rho$ denotes the radius of the desired circle, and $\psi_{k} \in \mathbb{R}^{2}$ is a unit vector on the line passing through agent $k$ and the target; that is,

$$
\psi_{k}(t)=\frac{p_{k}(t)-c_{k}(t)}{\left\|p_{k}(t)-c_{k}(t)\right\|} .
$$




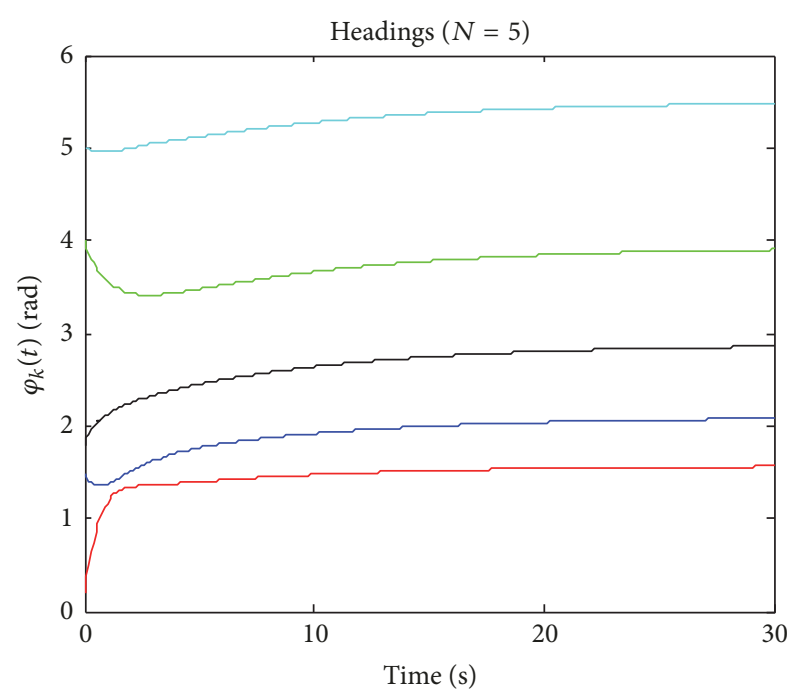

(a)

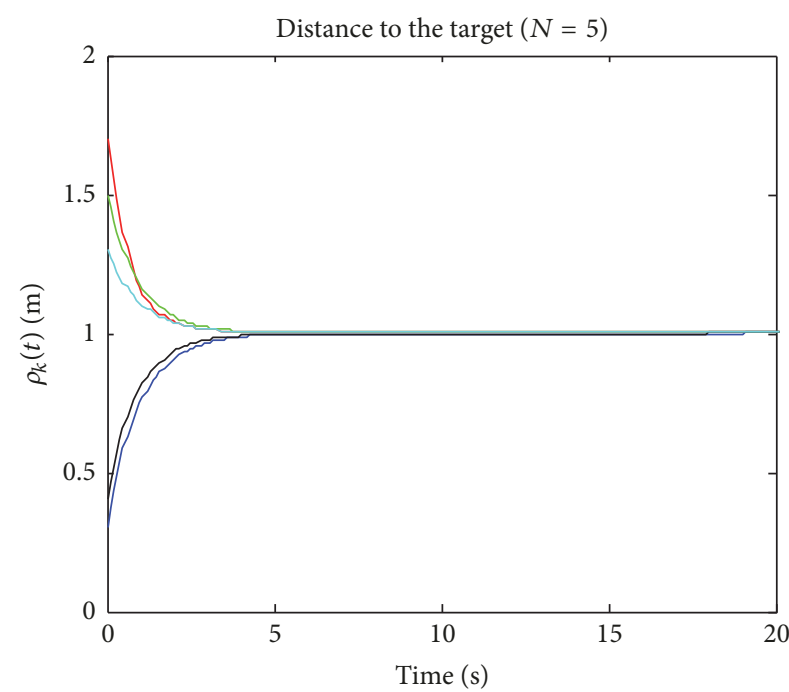

(b)

Figure 8: Trajectories with time of Figure 7. (a) Phases $\varphi_{k}(t)$ of agents. (b) Distances to the target.

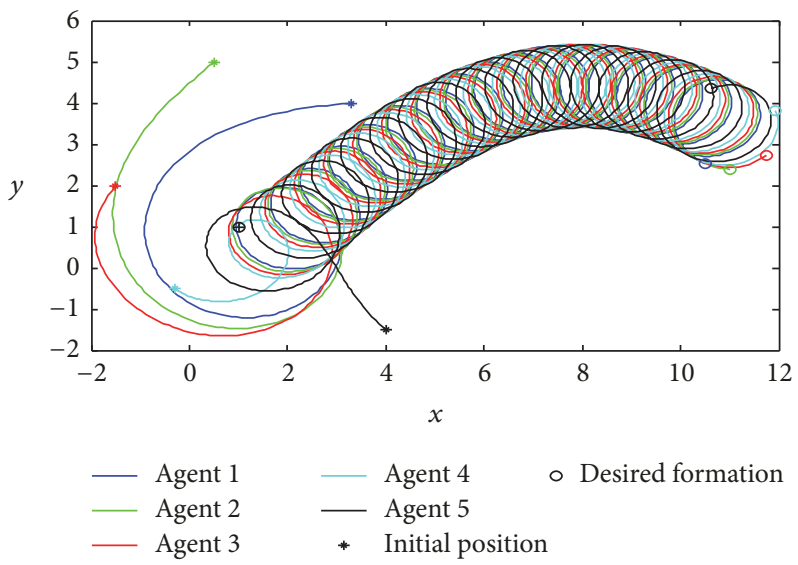

Figure 9: Circular formation trajectories $p_{k}(t)$ with a moving target.

2.2. Interaction Graphs. The communication topology is a connected ring topology in which each node is connected to two neighboring nodes. Each agent can only sense the relative positions of its two neighboring agents that are immediately in front of or behind it. Then, the graph describing the neighbor relationships is a weighted communication topology graph (undirected ring) $\mathscr{G}=(\mathscr{I}, \mathscr{E}, \mathscr{A})$, where $\mathscr{I}=$ $\{1,2, \ldots, N\}$ is the set of vertices, $\mathscr{E}=\{(1,2),(2,3), \ldots,(N-$ $1, N),(N, 1)\} \subseteq \mathscr{I} \times \mathscr{I}$ is the set of edges, and $\mathscr{A}=\left[a_{k j}\right]$ is the $N \times N$ adjacency matrix given by $a_{k j} \neq 0$ if $(k, j) \in \mathscr{E}$ and $a_{k j}=0$ otherwise. Both the adjacency and the Laplacian matrices of the ring topology are circulant; that is, they are completely defined by their first row; each subsequent row of the circulant matrix is the previous row shifted one position to the right with the first entry equal to the last entry of the previous row.

For a node $k \in \mathscr{I}$, the set of adjacent nodes is denoted by $\mathcal{N}_{k}$, which is called the neighbors of agent $k$. Agent $k$ 's two neighbors are denoted by $k^{+}$and $k^{-}$, which are labeled counterclockwise, by the following two rules:

$$
\begin{aligned}
& k^{+}= \begin{cases}k+1 & \text { when } k=1,2, \ldots, N-1 \\
1 & \text { when } k=N,\end{cases} \\
& k^{-}= \begin{cases}N & \text { when } k=1 \\
k-1 & \text { when } k=2,3, \ldots, N .\end{cases}
\end{aligned}
$$

Furthermore, as shown in Figure 1, the relative phases (bearing measurements to target) from agent $k$ to its immediate counterclockwise and clockwise neighboring agents are, respectively, denoted by variables $\varphi_{k k^{+}}$and $\varphi_{k k^{-}}$as follows: 


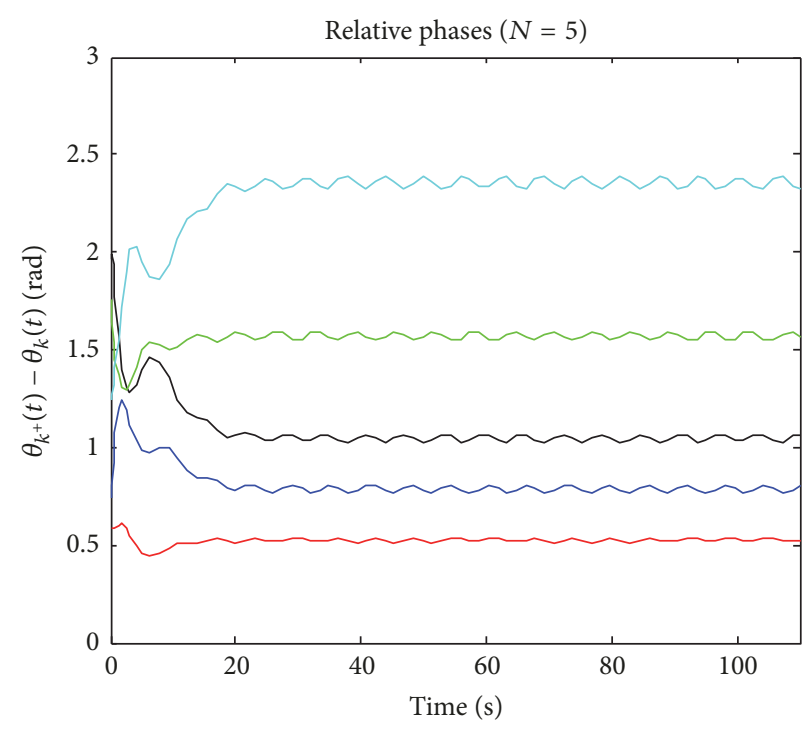

(a)

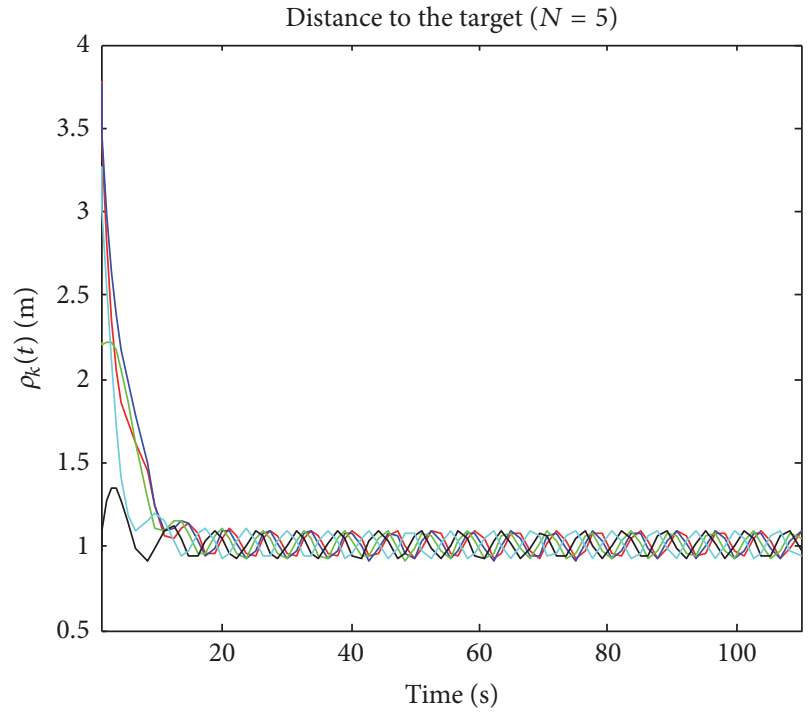

(b)

FIgURE 10: Trajectories with time of Figure 9. (a) Relative phases $\varphi_{k^{+} k}(t)$. (b) Distances to the target.

$$
\begin{aligned}
& \varphi_{k k^{+}}= \begin{cases}\varphi_{k}-\varphi_{k^{+}} & \text {when } k=1,2, \ldots, N-1 \\
P V\left(\varphi_{k}-\varphi_{k^{+}}\right) \triangleq\left[\left(\varphi_{k}-\varphi_{k^{+}}\right) \bmod 2 \pi\right]=\varphi_{k}-\varphi_{k^{+}}-2 \pi & \text { when } k=N,\end{cases} \\
& \varphi_{k k^{-}}= \begin{cases}\varphi_{k}-\varphi_{k^{-}} & \text {when } k=2,3, \ldots, N \\
P V\left(\varphi_{k}-\varphi_{k^{-}}\right) \triangleq\left[\left(\varphi_{k}-\varphi_{k^{-}}\right) \bmod 2 \pi\right]=\varphi_{k}-\varphi_{k^{-}}+2 \pi & \text { when } k=1 .\end{cases}
\end{aligned}
$$

Moreover, $\sum_{k=1}^{N}\left|\varphi_{k k^{+}}(t)\right| \equiv 2 \pi$ and $\sum_{k=1}^{N}\left|\varphi_{k k^{-}}(t)\right| \equiv 2 \pi$ always hold. The desired relative phases are denoted by $\varphi_{k k^{+}}^{*}$ and $\varphi_{k k^{-}}^{*}$. $\sum_{k=1}^{N}\left|\varphi_{k k^{+}}^{*}(t)\right| \equiv 2 \pi$ and $\sum_{k=1}^{N}\left|\varphi_{k k^{-}}^{*}(t)\right| \equiv 2 \pi$ always hold as well.

Assumption 1. No two agents initially occupy the same phase, and the initial phases $\varphi_{k}\left(t_{0}\right)$ satisfy

$$
\begin{aligned}
0 & \leq \varphi_{1}\left(t_{0}\right)<\varphi_{2}\left(t_{0}\right)<\cdots<\varphi_{k}\left(t_{0}\right)<\varphi_{k+1}\left(t_{0}\right)<\cdots \\
& <\varphi_{N}\left(t_{0}\right)<2 \pi .
\end{aligned}
$$

2.3. Control Objectives. The main objective is to design a distributed control law $u_{k}(t)$ such that the agents in (1) are formulated to a circular motion in any preset desired pattern (not limited to uniform distribution) along the circle; moreover, the agents' spatial ordering can be preserved. The structure of feedback control design of circular formation is shown in Figure 2.

\section{Circular Motion Control Design}

In this section, a trajectory tracking control strategy is designed to stabilize an agent to a circular motion, as illustrated in the circular motion tracking control part of Figure 2.

3.1. Circling a Fixed Target. In this case, the control objective can be expressed as

$$
\lim _{t \rightarrow \infty} \tilde{p}_{k}(t)=R_{\theta_{k}}(t) \psi_{k}\left(t_{0}\right) \rho
$$

where $R_{\theta_{k}}(t)$ is the rotation matrix associated with $\theta_{k}(t)$ as

$$
R_{\theta_{k}}(t)=\left(\begin{array}{cc}
\cos \theta_{k}(t) & -\sin \theta_{k}(t) \\
\sin \theta_{k}(t) & \cos \theta_{k}(t)
\end{array}\right) .
$$

The right side of (6) represents a trajectory rotating on a circle with the radius $\rho$ and rotating direction decided by $R_{\theta_{k}}(t)$, which can be considered as the reference trajectory for the agent state. The tracking error is defined by $e_{k}(t)=\widetilde{p}_{k}(t)-$ $R_{\theta_{k}}(t) \psi_{k}\left(t_{0}\right) \rho$, and the control law is given by

$$
\begin{aligned}
u_{k}(t)= & -\beta\left(\tilde{p}_{k}(t)-R_{\theta_{k}}(t) \psi_{k}\left(t_{0}\right) \rho\right) \\
& +\dot{R}_{\theta_{k}}(t) \psi_{k}\left(t_{0}\right) \rho,
\end{aligned}
$$


where $\beta>0$ is the control parameter and

$$
\dot{R}_{\theta_{k}}(t)=\dot{\theta}_{k}(t)\left(\begin{array}{cc}
-\sin \theta_{k}(t) & -\cos \theta_{k}(t) \\
\cos \theta_{k}(t) & -\sin \theta_{k}(t)
\end{array}\right) \text {. }
$$

Theorem 2. Consider a once differentiable function $\theta_{k}(t)$ with a bounded first time derivative; under the control law (8), $\widetilde{p}_{k}(t)$ asymptotically converges to $R_{\theta_{k}}(t) \psi_{k}\left(t_{0}\right) \rho$ for any initial condition $p_{k}\left(t_{0}\right)$, meaning that agent $k$ is enforced to converge to a circular motion of center $c$ and radius $\rho$ by tracking $a$ rotating trajectory with direction determined by the sign of $\dot{\theta}_{k}(t)$.

Proof. Differentiating the tracking error $e_{k}(t)$ with respect to time and applying the control law (8), one has

$$
\begin{aligned}
\dot{e}_{k}(t) & =\dot{p}_{k}(t)-\dot{R}_{\theta_{k}}(t) \psi_{k}\left(t_{0}\right) \rho \\
& =u_{k}(t)-\dot{R}_{\theta_{k}}(t) \psi_{k}\left(t_{0}\right) \rho \\
& =-\beta\left(\tilde{p}_{k}(t)-R_{\theta_{k}}(t) \psi_{k}\left(t_{0}\right) \rho\right)=-\beta e_{k}(t) .
\end{aligned}
$$

Therefore, the tracking error $e_{k}(t)$ asymptotically converges to zero. Consider the given $\widetilde{p}_{k}(t)$ and $\dot{\theta}_{k}(t)$; the closedloop dynamics of the original system will formulate a circular motion with tracking a rotating trajectory.

Remark 3. From both sides of (6), we note that the initial direction of $\widetilde{p}_{k}(t)$ and $R_{\theta_{k}}(t) \psi_{k}\left(t_{0}\right) \rho$ is the same. Therefore, if a uniform angular velocity $\dot{\theta}_{k}(t)=\omega \neq 0$ is adopted, a circular motion with initial phase arrangement can be achieved.

3.2. Circling without Target. Instead of circling a target, now we consider the problem of how the agents are formulated to circular formation with circling the resulting coincident center. A protocol for center consensus can be firstly designed as

$$
\dot{c}_{k}(t)=-k_{c} \sum_{j \in \mathscr{N}_{k}(t)} a_{k j}(t)\left(c_{k}(t)-c_{j}(t)\right)
$$

where $k_{c}>0$. Equation (11) can also be written in a vector form as

$$
\dot{C}(t)=-k_{c} L(t) \otimes I_{2} C(t)
$$

where $C(t)=\left[c_{1}(t), \ldots, c_{N}(t)\right]^{T}$ and $L(t)$, which represents the communication topology, is bounded and piecewise continuous in time.

Lemma 4 (see [29]). Suppose that $\mathscr{G}(t)=(\mathscr{I}, \mathscr{E}(t), \mathscr{A}(t))$ is uniformly connected and $L(t)$ is bounded and piecewise continuous in time. Then, the equilibrium set $\bar{C}=\{C \in$ $\left.\mathbb{R}^{2 N}: c_{k}=c_{j} \forall k, j \in \mathscr{I}\right\}$ of (12) is uniformly exponentially stable. Furthermore, the states converge to a center point in $\bar{C}=[\bar{c}, \ldots, \bar{c}]^{T}$.

Consider the center $c_{k}(t)$ to be a time-varying variable here; the tracking error is redefined by $e_{k}(t)=\widetilde{p}_{k}(t)-$ $R_{\theta_{k}}(t) \psi_{k}(t) \rho$, where time-varying $c_{k}(t)$ replaces the fixed target $c$ in $\widetilde{p}_{k}(t)$ and $\psi_{k}(t)$. Then, the error dynamics can be expressed as follows:

$$
\begin{aligned}
\dot{e}_{k}(t)= & \dot{p}_{k}(t)-\dot{c}_{k}(t)-\dot{R}_{\theta_{k}}(t) \psi_{k}(t) \rho \\
& -R_{\theta_{k}}(t) \dot{\psi}_{k}(t) \rho \\
= & u_{k}(t)-\dot{c}_{k}(t)-\dot{R}_{\theta_{k}}(t) \psi_{k}(t) \rho \\
& -R_{\theta_{k}}(t) \dot{\psi}_{k}(t) \rho .
\end{aligned}
$$

And a circular motion control law for the agents can be given as

$$
\begin{aligned}
u_{k}(t)= & -\beta\left(\left(p_{k}(t)-c_{k}(t)\right)-R_{\theta_{k}}(t) \psi_{k}(t) \rho\right) \\
& +\dot{R}_{\theta_{k}}(t) \psi_{k}(t) \rho+R_{\theta_{k}}(t) \dot{\psi}_{k}(t) \rho+\dot{c}_{k}(t) .
\end{aligned}
$$

Theorem 5. Consider a once differentiable function $\theta_{k}(t)$ with bounded first time derivative; under the center consensus law (12) and the control law (14), $\bar{e}_{k}(t)$ converges to zero for any initial conditions $p_{k}\left(t_{0}\right)$ and $c_{k}\left(t_{0}\right)$; that is, all agents are simultaneously enforced to converge to a common circle of center $\bar{c}$ and radius $\rho$ by tracking a rotating transformed trajectory with direction determined by the sign of $\dot{\theta}_{k}(t)$.

Proof. Lemma 4 confirms the existence of $\bar{c}$ such that $\lim _{t \rightarrow \infty} c_{k}(t)=\bar{c}$ for all $k \in \mathscr{I}$. Based on the existence of $\bar{c}$, a new tracking error $\bar{e}_{k}(t)$ is defined as $\bar{e}_{k}(t)=\left(p_{k}(t)-\right.$ $\bar{c})-R_{\theta_{k}}(t) \bar{\psi}_{k} \rho$, where $\bar{c}$ replaces $c_{k}(t)$ in $\bar{\psi}_{k}$. Differentiating the tracking error $\bar{e}_{k}(t)$ with respect to time and applying the control law (14), one has

$$
\begin{aligned}
\dot{\bar{e}}_{k}(t) & =\dot{p}_{k}(t)-\dot{R}_{\theta_{k}}(t) \bar{\psi}_{k} \rho=u_{k}(t)-\dot{R}_{\theta_{k}}(t) \psi_{k}\left(t_{0}\right) \rho \\
& =-\beta \bar{e}_{k}(t) \underbrace{-\beta e_{c_{k}}(t)-e_{\psi_{k}}(t)\left(\beta R_{\theta_{k}}(t) \rho+\dot{R}_{\theta_{k}}(t) \rho\right)+R_{\theta_{k}}(t) \dot{\psi}_{k}(t) \rho+\dot{c}_{k}(t)}_{w(t) \triangleq}=-\beta \bar{e}_{k}(t)+w(t),
\end{aligned}
$$

where $e_{c_{k}}(t)=\bar{c}-c_{k}(t)$ and $e_{\psi_{k}}(t)=\bar{\psi}_{k}-\psi(t)$. Thus, the overall error dynamics can be written in a vector form as

$$
\dot{\bar{E}}(t)=-\beta \bar{E}(t)+W(t)
$$

where $\bar{E}(t)=\left[\bar{e}_{1}(t), \ldots, \bar{e}_{N}(t)\right]^{T}$ and $W(t)=$ $\left[w_{1}(t), \ldots, w_{N}(t)\right]^{T}$. Note that (16) and the dynamics of $W(t)$ form a cascade system. It is noted from Lemma 4 that $e_{c_{k}}(t), e_{\psi_{k}}(t), \dot{\psi}_{k}$, and $\dot{c}_{k}(t)$ converge to zero; that is, $W(t)=0$, 
as $t \rightarrow \infty$. Thus, $\bar{e}_{k}(t)$ will converge to zero asymptotically according to $\dot{\bar{e}}_{k}(t)=-\beta \bar{e}_{k}(t)$.

Remark 6. Note that the center consensus subsystem and the circle control subsystem form a cascade system such that the center consensus subsystem does not rely on the position information. Thus, before the system starts, each agent is initialized at any random center instead of a real target, and all centers can reach a common center point far faster than that of the formation control.

\section{Any Preset Phase Arrangement along a Circle}

The previous control law does not take into consideration the phase configuration problem among agents. In order to stabilize the agents to a circular formation with any preset phase distribution, the circular motion control law (8) or (14) must include a cooperative part for arranging phases.
Through the analysis of control law (8) or (14), the rotation matrix $R_{\theta_{k}}(t)$ is found as a breakthrough point. The phase $\varphi_{k}(t)$ of agent $k$ can be arranged by controlling the rotation angle $\theta_{k}(t)$ of $R_{\theta_{k}}(t)$ with the relative phases $\varphi_{k k^{+}}$and $\varphi_{k k^{-}}$. Thus, a control scheme is designed as shown in the phase control part of Figure 2.

Firstly, a phase control law has been proposed in [21] as

$$
\begin{aligned}
\dot{\varphi}_{k}(t)= & -\frac{\left|\varphi_{k k^{-}}^{*}\right|}{\left|\varphi_{k k^{+}}^{*}\right|+\left|\varphi_{k k^{-}}^{*}\right|} \varphi_{k k^{+}}(t) \\
& -\frac{\left|\varphi_{k k^{+}}^{*}\right|}{\left|\varphi_{k k^{+}}^{*}\right|+\left|\varphi_{k k^{-}}^{*}\right|} \varphi_{k k^{-}}(t) \quad k=1,2, \ldots, N .
\end{aligned}
$$

With the stacked column vector $\Phi(t)$ = $\left[\varphi_{1}(t), \varphi_{2}(t), \ldots, \varphi_{N}(t)\right]^{T}$, (17) can be written in a vector form as
$\dot{\Phi}(t)=-L^{*} \Phi(t)$

$$
L^{*}=\left[\begin{array}{cc}
1 & -\frac{\left|\varphi_{1 N}^{*}\right|}{\left|\varphi_{12}^{*}\right|+\left|\varphi_{1 N}^{*}\right|} \\
-\frac{\left|\varphi_{23}^{*}\right|}{\left|\varphi_{23}^{*}\right|+\left|\varphi_{21}^{*}\right|} & 1 \\
0 & -\frac{\left|\varphi_{34}^{*}\right|}{\left|\varphi_{34}^{*}\right|+\left|\varphi_{32}^{*}\right|} \\
\vdots & \vdots \\
0 & 0 \\
-\frac{\left|\varphi_{N 1}^{*}\right|}{\left|\varphi_{N 1}^{*}\right|+\left|\varphi_{N(N-1)}^{*}\right|} & 0
\end{array}\right.
$$

Lemma 7 (see [21]). Given any admissible circular formation characterized by $\varphi_{k k^{+}}^{*}$ and $\varphi_{k k^{-}}^{*}$, the circular formation problem is solved with order preservation under the proposed control law (17).

The above phase control law (17) can drive the phase $\varphi_{k}(t)$ of agent $k$ to move towards its waypoint that is determined completely by its two neighbors' relative phases and the desired relative phases $\varphi_{k k^{+}}^{*}$ and $\varphi_{k k^{-}}^{*}$. Instead of a fixed circular formation, in order to keep the phase arrangement always in a circular motion, a new waypoint based phase control law can be equivalently redesigned in a rotating manner as

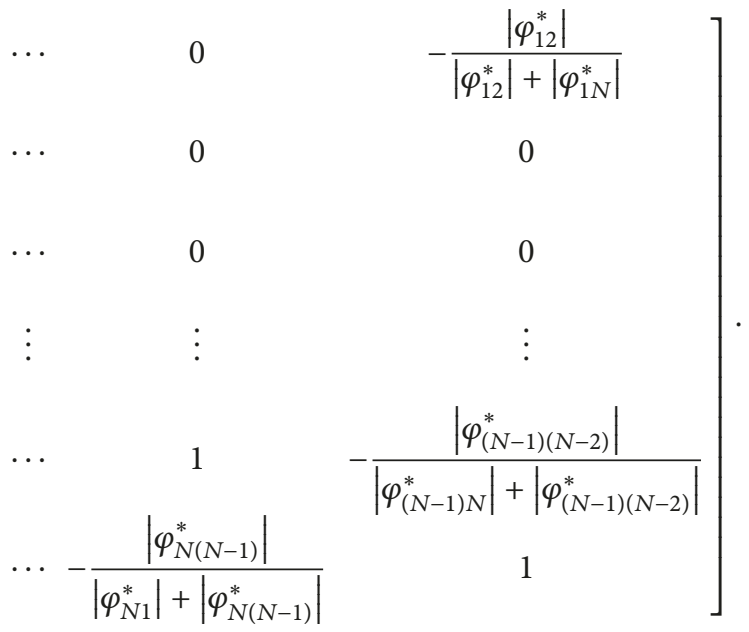

$$
\left.-\frac{\left|\varphi_{k k^{+}}^{*}\right|}{\left|\varphi_{k k^{+}}^{*}\right|+\left|\varphi_{k k^{-}}^{*}\right|} \varphi_{k k^{-}}(t)\right)
$$

where $\omega_{0}$ is a constant and $\alpha>0$ is a control parameter. One feature of the proposed control law (20) is that it guarantees that the spatial ordering of the agents is preserved throughout the system's evolution in the initial condition (5), and thus no collision takes place during the process of circular formation. In addition, it is noted that the relationship between the phase $\varphi_{k}(t)$ and the rotation angle $\theta_{k}(t)$ is

$$
\dot{\varphi}_{k}(t)=\omega_{0}+\alpha\left(-\frac{\left|\varphi_{k k^{-}}^{*}\right|}{\left|\varphi_{k k^{+}}^{*}\right|+\left|\varphi_{k k^{-}}^{*}\right|} \varphi_{k k^{+}}(t)\right.
$$

$$
\theta_{k}(t)=\varphi_{k}(t)-\varphi_{k}\left(t_{0}\right)
$$


Then, combined with the phase control, the control law (8) can be redesigned as

$$
\begin{aligned}
u_{k}(t)= & -\beta\left(\widetilde{p}_{k}(t)-R_{\theta_{k}}(t) \psi_{k}\left(t_{0}\right) \rho\right) \\
& +\dot{\varphi}_{k}(t) R_{\theta_{k}}(t) \psi_{k}\left(t_{0}\right) \rho,
\end{aligned}
$$

where $\dot{\varphi}_{k}(t)$ is given in (20).

Theorem 8. Let $\mathscr{G}$ be a ring topology graph and $L^{*}$ be its corresponding Laplacian matrix defined by (19). Consider model (1) with any initial conditions $p_{k}\left(t_{0}\right)$ and satisfying Assumption 1; under the circular motion control law (22) and phase control law (20), the agents are all enforced to converge to a circular formation of center $c$ and radius $\rho$ with phase arrangement by presetting $\varphi_{k k^{+}}^{*}$ and $\varphi_{k k^{-}}^{*}$, for $k=1,2, \ldots, N$. Simultaneously, the agents' spatial ordering is preserved.

Proof. The proof of the circular motion control part is analyzed in Theorem 2 . The convergence proof of the phase control part is a consequence of Theorem 1 of [21]. So, the proof of this theorem is omitted here.

In some practical situations, the uneven distribution circular formation needs some particular agents to observe the target from particular directions, such as agent 1 . In this case, by introducing a phase reference $\varphi_{d}$, the phase control law for this agent can be redesigned as

$$
\begin{aligned}
\dot{\varphi}_{1}(t) & =\omega_{0}+\alpha\left(-\frac{\left|\varphi_{1 N}^{*}\right|}{\left|\varphi_{12}^{*}\right|+\left|\varphi_{1 N}^{*}\right|} \varphi_{12}(t)\right. \\
& \left.-\frac{\left|\varphi_{12}^{*}\right|}{\left|\varphi_{12}^{*}\right|+\left|\varphi_{1 N}^{*}\right|} \varphi_{1 N}(t)\right)+d \sin \left(\varphi_{d}-\varphi_{1}\right),
\end{aligned}
$$

where $d>0$ is a constant. This yields the following extension of Theorem 8 .

Corollary 9. Under the same conditions of Theorem 8, with the circular motion control law (22) and phase control laws (20) and (23), all the agents will be enforced to converge to a circular formation as described in Theorem 8 and the phases $\varphi_{k}$ of agent $k$ can be steered to their desired phase $\varphi_{k}^{*}$ for $k=2, \ldots, N$ by referring to the phase $\varphi_{1}=\varphi_{d}$ of agent 1 .

Proof. By defining $y_{k}(t)=\varphi_{k^{+} k}(t)$, the stability of the control system can be studied by Lyapunov function as

$$
V=\frac{1}{2} y^{T} y+d\left(1-\cos \left(\varphi_{d}-\varphi_{1}\right)\right)
$$

where $y=\left[y_{1}, y_{2}, \ldots, y_{N}\right]^{T}$. Differentiating $V$ yields

$$
\dot{V}=-\alpha y^{T} L^{*} y+d \sin \left(\varphi_{d}-\varphi_{1}\right)\left(\dot{\varphi}_{d}-\dot{\varphi}_{1}\right) .
$$

The control law (23) with $\dot{\varphi}_{d}=\omega_{0}$ for agent 1, (20) for agents $k=2, \ldots, N$, and $u_{k}$ given by (22) result in $\dot{V}=$ $-\alpha y^{T} L^{*} y \leq 0$ and $L^{*} \Phi(t)=0$ because the matrix $L^{*}$ has rank $N-1$. The relation

$$
d \sin \left(\varphi_{d}-\varphi_{1}\right)=0
$$

implies that $\varphi_{1}=\varphi_{d}$ or $\varphi_{d}+\pi$. Therefore, the relative equilibrium is $\varphi_{1}=\varphi_{d}$ which minimizes the potential $V$.
It is noted that, in the case $\omega_{0}=0$, Corollary 9 proves that the agents are stabilized to a fixed circular formation with agent 1's phase $\varphi_{1}=\varphi_{d}$, so that the other agents are localized with respect to agent 1's position. In addition, the result of Theorem 8 and Corollary 9 can also be applied to the timevarying center case; that is, the control law (14) can also be combined with phase control law (23).

\section{Simulation Results}

This section presents the simulation of five agents $(N=$ 5) modeled by (1), governed by the proposed control strategies above. The desired circular formation is defined by radius $\rho=1$ and relative headings are arranged as $\pi / 6, \pi / 4, \pi / 3, \pi / 2,3 \pi / 4$. The simulation results with $k_{c}=$ $\alpha=\beta=\omega_{0}=d=1$ are, respectively, demonstrated in the following several scenarios. (1) Figures 3 and 4 show that all the agents circumnavigate the fixed target $c=(1,1)$ under the circular motion control law (22) and the phase control law (20). (2) While there is no fixed target, the control laws (14) and (20) formulate a circle circumnavigating the resulting coincident center by the consensus law (11) as shown in Figures 5 and 6. (3) In Figures 7 and 8, a fixed circular formation for positioning agent 1 at $\varphi_{d}=\pi / 2$ is formulated by the control laws (20), (22), and (23). (4) All the agents circumnavigate a slow moving target under the control laws (14) and (20) as shown in Figures 9 and 10, where $c(t)=$ $(1+(1 / 10) t, 1+4.5 \sin (2 \pi / 300) t)^{T}$.

The First Scenario. See Figures 3 and 4.

The Second Scenario. See Figures 5 and 6.

The Third Scenario. See Figures 7 and 8.

The Fourth Scenario. See Figures 9 and 10.

All the above simulation results show that the group of agents can asymptotically converge to the desired circular formation under the proposed control law. In particular, the figures clearly demonstrate that the agents preserve their orderings in the entire motion.

\section{Conclusion}

A new control strategy is presented to formulate a desired circular formation with any preset phase distribution. The problem is solved in two parts: circular motion control and phase control. The circular motion control part is designed to circumnavigate a fixed target. Particularly, with a center consensus method, no target case is solved by considering the center $c_{k}(t)$ to be a time-varying variable. Through combining the circular motion control law with a phase control law, the limitation on a one-dimensional space of circular motion in [21] is released by introducing the radius control of circular motion. Furthermore, the positioning problem of agents on the circle is solved for some application situations. Future works will focus on extending the proposed strategy to agents with more practical dynamics, including some additional 
constraints to ensure obstacle avoidance. In addition, it is of interest to consider the extension of the proposed strategy to three-dimensional space.

\section{Conflicts of Interest}

The authors declare that they have no conflicts of interest.

\section{Acknowledgments}

This work was supported by the Scientific Research Fund of Liaoning Provincial Education Department (L2016011), Liaoning Provincial Natural Science Foundation (201601321), and Talent Scientific Research Fund of LSHU (2016XJJ-033) of China.

\section{References}

[1] Z. Peng, D. Wang, Y. Shi, H. Wang, and W. Wang, "Containment control of networked autonomous underwater vehicles with model uncertainty and ocean disturbances guided by multiple leaders," Information Sciences, vol. 316, pp. 163-179, 2015.

[2] B. Das, B. Subudhi, and B. B. Pati, "Co-operative control coordination of a team of underwater vehicles with communication constraints," Transactions of the Institute of Measurement and Control, vol. 38, no. 4, pp. 463-481, 2016.

[3] X. Dong, B. Yu, Z. Shi, and Y. Zhong, “Time-varying formation control for unmanned aerial vehicles: theories and applications," IEEE Transactions on Control Systems Technology, vol. 23, no. 1, pp. 340-348, 2015.

[4] R. Olfati-Saber, J. A. Fax, and R. M. Murray, "Consensus and cooperation in networked multi-agent systems," Proceedings of the IEEE, vol. 95, no. 1, pp. 215-233, 2007.

[5] N. E. Leonard, D. A. Paley, F. Lekien, R. Sepulchre, D. M. Fratantoni, and R. E. Davis, "Collective motion, sensor networks, and ocean sampling," Proceedings of the IEEE, vol. 95, no. 1, pp. 4874, 2007.

[6] R. Olfati-Saber and R. M. Murray, "Consensus problems in networks of agents with switching topology and time-delays," Institute of Electrical and Electronics Engineers Transactions on Automatic Control, vol. 49, no. 9, pp. 1520-1533, 2004.

[7] D. A. Paley, N. E. Leonard, and R. Sepulchre, "Stabilization of symmetric formations to motion around convex loops," Systems \& Control Letters, vol. 57, no. 3, pp. 209-215, 2008.

[8] M. Ou, H. Du, and S. Li, "Finite-time formation control of multiple nonholonomic mobile robots," International Journal of Robust and Nonlinear Control, vol. 24, no. 1, pp. 140-165, 2014.

[9] R. Olfati-Saber, "Flocking for multi-agent dynamic systems: algorithms and theory," Institute of Electrical and Electronics Engineers Transactions on Automatic Control, vol. 51, no. 3, pp. 401-420, 2006.

[10] P. Ogren, E. Fiorelli, and N. E. Leonard, "Cooperative control of mobile sensor networks: adaptive gradient climbing in a distributed environment," IEEE Transactions on Automatic Control, vol. 49, no. 8, pp. 1292-1302, 2004.

[11] D. V. Dimarogonas and K. J. Kyriakopoulos, "On the rendezvous problem for multiple nonholonomic agents," Institute of Electrical and Electronics Engineers Transactions on Automatic Control, vol. 52, no. 5, pp. 916-922, 2007.
[12] W. Wu and F. Zhang, "A speeding-up and slowing-down strategy for distributed source seeking with robustness analysis," IEEE Transactions on Control of Network Systems, vol. 3, no. 3, pp. 231-240, 2016.

[13] S. Li, R. Kong, and Y. Guo, "Cooperative distributed source seeking by multiple robots: Algorithms and experiments," IEEE/ASME Transactions on Mechatronics, vol. 19, no. 6, pp. 1810-1820, 2014.

[14] J.-C. Juang, "On the formation patterns under generalized cyclic pursuit," Institute of Electrical and Electronics Engineers Transactions on Automatic Control, vol. 58, no. 9, pp. 2401-2405, 2013.

[15] L. Ma and N. Hovakimyan, "Vision-based cyclic pursuit for cooperative target tracking," Journal of Guidance, Control, and Dynamics, vol. 36, no. 2, pp. 617-622, 2013.

[16] C. Song, L. Liu, and G. Feng, "Coverage control for mobile sensor networks with input saturation," Unmanned Systems, vol. 4, no. 1, pp. 15-21, 2016.

[17] Y. J. Shi, R. Li, and K. L. Teo, "Cooperative enclosing control for multiple moving targets by a group of agents," International Journal of Control, vol. 88, no. 1, pp. 80-89, 2015.

[18] I. Shames, S. Dasgupta, B. Fidan, and B. D. Anderson, "Circumnavigation using distance measurements under slow drift," IEEE Transactions on Automatic Control, vol. 57, no. 4, pp. 889-903, 2012.

[19] L. Briñón-Arranz, L. Schenato, and A. Seuret, "Distributed source seeking via a circular formation of agents under communication constraints," IEEE Transactions on Control of Network Systems, vol. 3, no. 2, pp. 104-115, 2016.

[20] J. A. Marshall, M. E. Broucke, and B. A. Francis, "Formations of vehicles in cyclic pursuit," IEEE Transactions on Automatic Control, vol. 49, no. 11, pp. 1963-1974, 2004.

[21] S. Daingade, A. Sinha, A. V. Borkar, and H. Arya, "A variant of cyclic pursuit for target tracking applications: theory and implementation," Autonomous Robots, vol. 40, no. 4, pp. 669686, 2016.

[22] R. Zheng, Y. Liu, and D. Sun, "Enclosing a target by nonholonomic mobile robots with bearing-only measurements," Automatica, vol. 53, pp. 400-407, 2015.

[23] A. Jain and D. Ghose, "Collective circular motion in synchronized and balanced formations with second-order rotational dynamics," Communications in Nonlinear Science and Numerical Simulation, vol. 54, pp. 156-173, 2018.

[24] Z. Chen and H.-T. Zhang, "No-beacon collective circular motion of jointly connected multi-agents," Automatica, vol. 47, no. 9, pp. 1929-1937, 2011.

[25] Z. Chen and H.-T. Zhang, "A remark on collective circular motion of heterogeneous multi-agents," Automatica, vol. 49, no. 5, pp. 1236-1241, 2013.

[26] R. Sepulchre, D. A. Paley, and N. E. Leonard, "Stabilization of planar collective motion: all-to-all communication," IEEE Transactions on Automatic Control, vol. 52, no. 5, pp. 811-824, 2007.

[27] C. Wang, G. Xie, and M. Cao, "Forming circle formations of anonymous mobile agents with order preservation," IEEE Transactions on Automatic Control, vol. 58, no. 12, pp. 32483254, 2013.

[28] C. Wang, G. Xie, and M. Cao, "Controlling anonymous mobile agents with unidirectional locomotion to form formations on a circle," Automatica, vol. 50, no. 4, pp. 1100-1108, 2014. 
[29] L. Moreau, "Stability of continuous-time distributed consensus algorithms," in Proceedings of the 43rd IEEE Conference on Decision and Control (CDC '04), pp. 3998-4003, December 2004. 


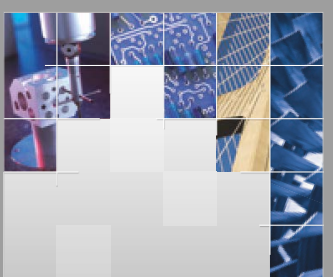

\section{Enfincering}
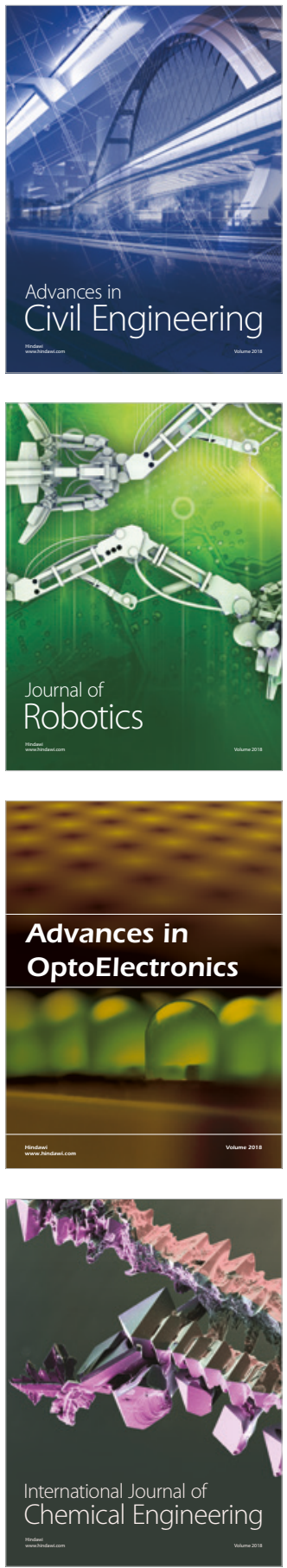

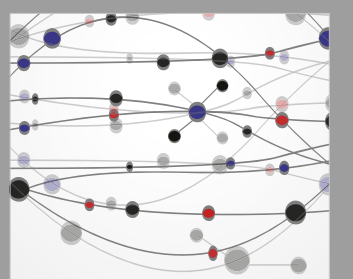

\section{Rotating \\ Machinery}

The Scientific World Journal

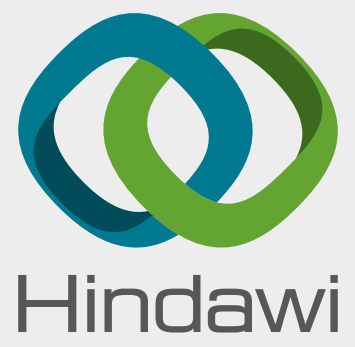

Submit your manuscripts at

www.hindawi.com
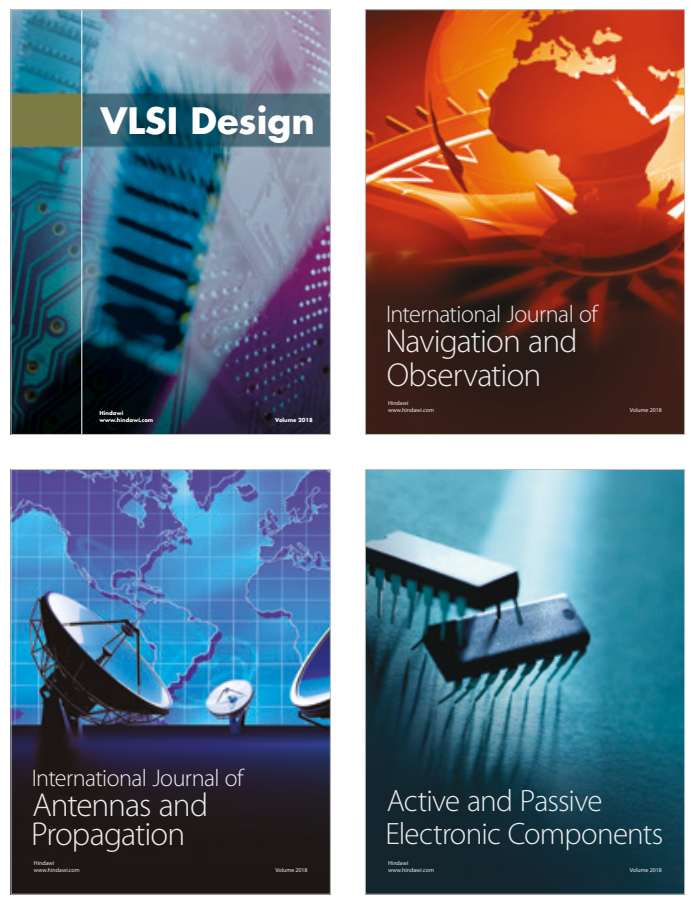
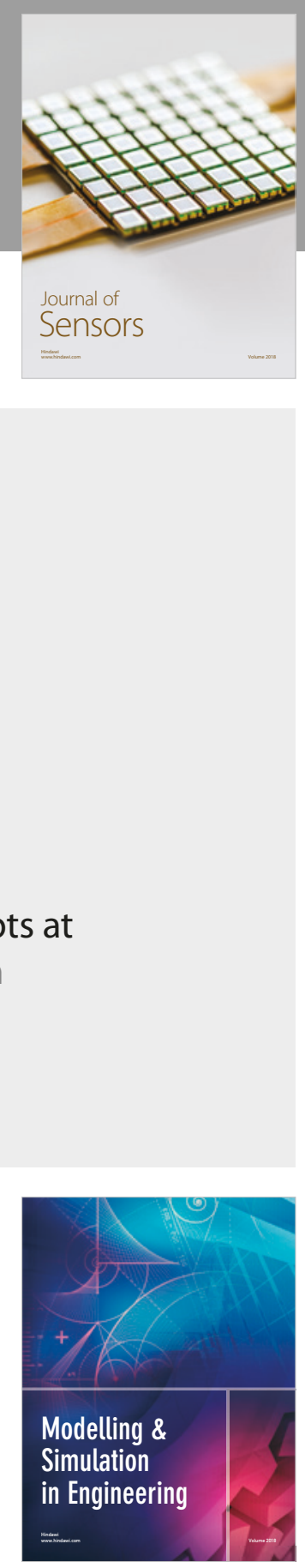

\section{Advances \\ Multimedia}
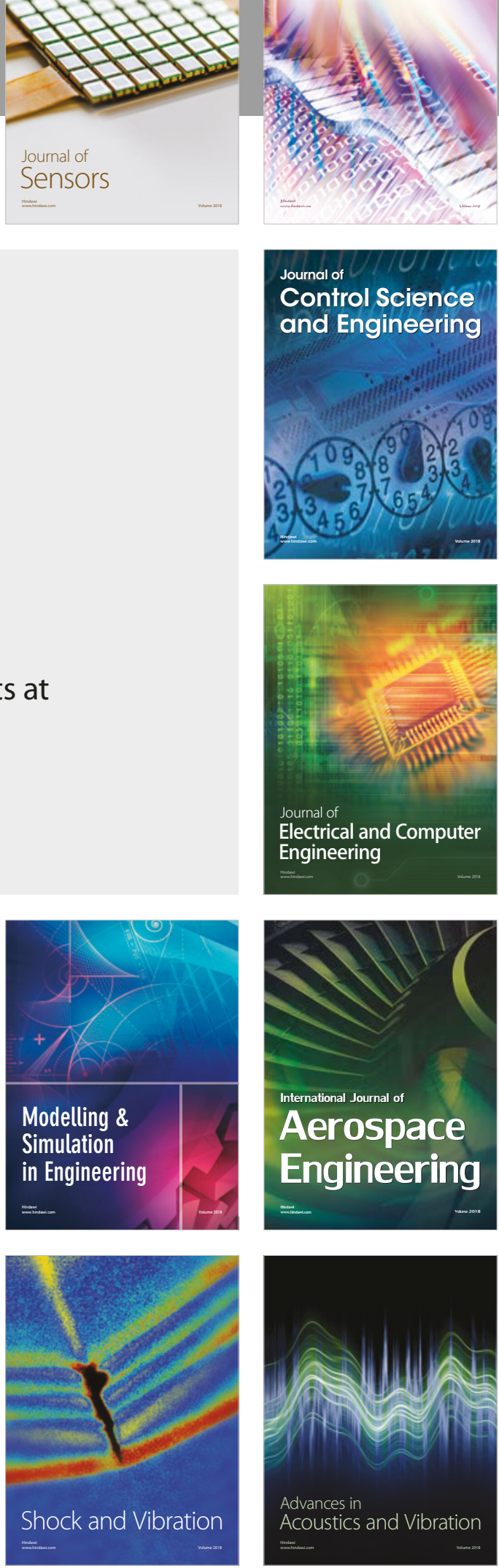